\title{
Regeneration of Fungiform Taste Buds: Temporal and Spatial Characteristics 1
}

\author{
MARYLOU CHEAL ${ }^{2}$ AND BRUCE OAKLEY \\ Division of Biological Sciences, The University of Michigan, Ann Arbor, \\ Michigan 48109
}

\begin{abstract}
The gross morphology of the tongue of the Mongolian gerbil Meriones unguiculatus), the location of papillae and taste buds, and the normal innervation pattern of the tongue and taste buds were determined. The chorda tympani nerve was interrupted to produce degeneration of fungiform taste buds. Regenerating chorda tympani axons followed the original nerve pathways in the tongue en route to the fungiform papillae in the epithelium where they initiated the regeneration of taste buds. The spatial distribution of reinnervated fungiform papillae and reformed taste buds was examined 7 to 19 days following surgery. Beginning at eight days following chorda tympani interruption there was a progressive increase, first, in the proportion of fungiform papillae that were reinnervated, and later in the number of reformed taste buds. On the basis of these measures it was concluded that a taste bud is reformed one to two days after reinnervation of its papilla. From the time course of reinnervation of the fungiform papillae it was calculated that some fibers regenerated at rates in excess of $2 \mathrm{~mm} / \mathrm{day}$. Regeneration was precise and systematic. The regenerating chorda tympani fibers accurately returned to the fungiform papillae; they did not follow the pathways of lingual nerve axons. In the initial stages of recovery both reinnervated papillae and reformed taste buds were preferentially located toward the front of the tongue; the reinnervation of posterior fungiform papillae was delayed.
\end{abstract}

Mammalian lingual taste buds are pearshaped clusters of elongated epithelial cells. Chemical stimuli contact the receptor cells of the taste bud through a minute pore in the epithelial keratin. Sensory axons innervate, and receive excitation from, the receptor cells.

Taste buds are found in three different types of papillae on the mammalian tongue. The foliate and vallate papillae, which are located in the posterior part of the tongue, contain several hundred taste buds innervated by the IXth nerve. The fungiform papillae are widely distributed on the anterior two thirds of the tongue. Each fungiform papilla in the gerbil contains a single taste bud innervated by the chorda tympani nerve. See figure 1A.

One hundred years ago von Vintschgau and Honigschmied (1877) discovered that rabbit foliate taste buds degenerated when deprived of their nerve supply. In subsequent years this trophic dependence of tongue taste buds upon intact innervation has been repeatedly demonstrated in mammals (Olmstead, '21; Guth, '57; Farbman, '69; Fujimoto and Murray, '70; Vij et al., '72; State and Bowden, '74; Oakley, '74; El-Eishi and State, 74). Denervated taste buds degenerate at rates that vary with the species, the type of papilla, and the individual taste bud. Rat and rabbit vallate taste buds disappear within seven to ten days (Guth, '57; Conger and Wells, '69; Fujimoto and Murray, 70; State and Bowden, '74; El-Eishi and State, 74) whereas rabbit foliate taste buds do not disappear until 14 to 21 days after dener-

\footnotetext{
${ }^{1}$ Send reprint requests to: Bruce Oakley, Division of Biological Sciences, The University of Michigan, Ann Arbor, Michigan 48109 .

${ }^{2}$ Present Address: Neuropsychology Laboratory, McLean Hospital, Belmont, Massachusetts 02178.
} 
vation (Jeppsson, '69; Murray and Murray, '69; Iwayama, '70; State and Bowden, '74; El-Eishi and State, '74). Degeneration of fungiform taste buds has been examined only in the rat. In light microscopic studies rat fungiform taste buds disappeared within seven days after chorda tympani interruption (Beidler, '63). Electron microscopy revealed degeneration in nerve terminals three to six hours after denervation and the disappearance of most fungiform buds within seven days (Farbman, '69).

Many investigators have shown that taste buds will regenerate in adults if sufficient time is allowed for nerve fibers to reinnervate the taste papillae (Guth, '58; Oakley, '67; Zalewski, '69; Jeppsson, '69; Fujimoto and Murray, '70; Iwayama, '70; El-Eishi and State, '74). The distribution and number of regenerated taste buds is characteristic of the tongue region and the type of papillae and not of the cranial nerve origin of the taste fibers which innervate the taste buds (Oakley, '70).

When rabbit vallate papillae were examined, it was found that taste buds began to reappear two weeks after crushing of the IXth nerve and were present in approximately normal numbers after four weeks (El-Eishi and State, '74). Varying rates of reformation of rabbit foliate taste buds have been reported (El-Eishi and State, '74, 21 days; Fujimoto and Murray, '70, 25 days; Iwayama, '70, 40 days; Jeppsson, '69, 6 to 7 months). Some of this variation may result from the fact that in all studies except one (El-Eishi and State, '74) the IXth nerve was cut rather than crushed. Regeneration of fungiform taste buds has not been studied.

We carried out one study of chorda tympani nerve regeneration and a separate study of fungiform taste bud regeneration in gerbils. Silver staining allowed us to measure quantitatively both the time course of reappearance of nerve fibers in fungiform papillae and the location of reinnervated papillae. In other gerbils, we quantitatively measured the time course of fungiform taste bud reformation and the location of reformed taste buds using a hematoxylin stain. To minimize variability in regeneration time and maximize the proportion of axons which returned, we usually crushed, rather than cut, the chorda tympani nerve. The widely dispersed fungiform papillae are more suited to an analysis of the spatial pattern of regenerating axons and taste buds than are the compactly localized foliate or vallate papillae. We did not study taste bud degeneration.

\section{METHODS}

Eighty-five male and female Mongolian gerbils, Meriones unguiculatus, 3 to 7 months old, were used in this study. Experimental animals and breeding stock were obtained from a closed colony maintained by J. J. Hasenau of Northville, Michigan.

Operations to interrupt various nerves to the tongue were carried out under sodium pentobarbital anesthesia. The animals, weighing between 35 and $60 \mathrm{gm}$, received an initial dose of $2.5 \mathrm{mg}$ i.p., followed by 2 mg 15 minutes later. In chronic operations each animal received 5000 units of penicillin i.m.

The chorda tympani nerve, after it exits distally from the tympanic bulla, unites with the lingual nerve proper to form the common lingual nerve which enters the tongue. The chorda tympani and lingual nerves were exposed near the site of union by separating the internal pterygoid muscles from the anterior face of the tympanic bulla. At this site the chorda tympani was interrupted in two ways. In most animals it was crushed by 40 brief and 5 sustained pinches with the tips of \#5 jeweler's forceps at a single locus proximal to the union with the lingual nerve. In other experiments the chorda was cut through and left in situ without further disturbance. When the lingual, IXth or XIIth nerves were transected in control operations, $2-10 \mathrm{~mm}$ portions were removed to discourage regeneration. The IXth nerve was exposed near the posterior lacerated foramen and the XIIth nerve exposed near the posterior belly of the digastric muscle.

Gerbils were perfused with a mixture of 
$4 \%$ formaldehyde and $15 \%$ sucrose. Two histological procedures were used for light microscopic examination of tongues. Cross sections of paraffin embedded tongues were cut at $10 \mu \mathrm{m}$ and serial sections were stained with Heidenhain's iron hematoxylin to visualize taste buds. For study of normal and regenerating axons, sagittally cut 25$50 \mu \mathrm{m}$ serial frozen sections were stained with Winkelman's silver stain (Winkelman, '60). Since paraffin and frozen section procedures caused different amounts of shrinkage relative to the fresh tongue, the dimensions of all representations of the tongue in the figures have been adjusted to those of fresh tissue.

The normal pattern of tongue innervation and taste bud distribution has not been described previously for the gerbil. Consequently, the initial study consisted of determining the distribution of nerves and location of taste papillae by gross dissection of six fresh tongues. Additional detail on the distribution of the axons of the chorda tympani, IXth, or lingual plus chorda tympani was obtained by transecting in each case all other ipsilateral nerves and preparing the tongues for silver staining after ten days of survival. Normal taste bud appearance and locations were determined by hematoxylin staining of the tongues of five normal animals, and of 24 experimental animals in which one side of the tongue remained normally innervated. Finally, given that denervated taste buds degenerate, the source of sustaining nervous innervation of fungiform taste buds was determined by examining tongues in which the chorda tympani, or the IXth nerve, or both, had been transected.

After the appropriate times necessary for chorda tympani nerve regeneration and fungiform taste bud reformation were determined in pilot studies, the three principal experiments of this research were conducted.

First, silver staining was used to study reinnervation of fungiform papillae in 12 animals 7 to 11 days after the chorda tympani had been crushed unilaterally. For each histological section the position of axons within the epithelium was marked on a diagram of the tongue. The number of reinnervated papillae in each time period was calculated as a percentage of the normal number of fungiform papillae. Since denervated fungiform papillae were sometimes inconspicuous, we obtained the normal number by counting chorda tympani innervated fungiform papillae $(\bar{x}=53$, unilaterally) in each of three control animals that had the lingual, IXth, and XIIth nerves removed. The 12 experimental gerbils also had the ipsilateral IXth and lingual nerve proper transected. In addition six of these 12 animals had the ipsilateral XIIth nerve transected. The XIIth nerve was cut because we had some indication that it might send an occasional fiber to the dorsal epithelium. The lingual nerve was cut because it also sends fibers to the fungiform papillae. The IXth nerve is capable of reforming and functionally innervating fungiform papillae in the rat (Oakley, '67, '70). Consequently, the IXth nerve was removed as a control against unwanted collateral sprouting to the more distal portions of the tongue. Collateral sprouting by IXth nerve fibers was not detected in any experiments in this study.

In the second experiment taste buds were examined in 24 gerbils that had survived for 9 to 16 days after unilateral crushing of the chorda tympani nerve. In eight of these gerbils the ipsilateral IXth nerve was also transected. The tongues of five control operated gerbils (chorda tympani exposed but not touched) were also examined for taste buds. The number of regenerated taste buds revealed with hematoxylin stain was computed as a percentage of the number of fungiform taste buds on the intact side of the tongue.

In the third experiment the chorda tympani was unilaterally transected, rather than crushed, in nine gerbils. In six of these gerbils the ipsilateral IXth nerve was also transected. The gerbils were sacrificed 13 to 16 days later and the tongues stained for taste buds with iron hematoxylin.

Among all of the operated animals three had a weight loss greater than $5 \%$ in the 7 
to 16-day postoperative recovery period. Most animals gained weight.

\section{RESULTS}

\section{Observations on normal taste buds and innervation}

Taste buds on the gerbil tongue are found in the vallate, foliate, and fungiform papillae. Figure IA depicts these papillae in a dorsal view of a normal gerbil tongue. The vallate papilla has a trench or slit on each side of the midline of the tongue. The foliate papillae have 5 to 11 inconspicuous slits on the lateral edge of the tongue just anterior to the tongue's attachment to the buccal wall of the oral cavity. Frequently, there are unequal numbers of foliate slits on the left and right sides of a tongue. Taste buds line the trenches of the vallate and foliate papilla. Fungiform papillae are distributed on the dorsal surface of the anterior two thirds of the tongue. Each fungiform papilla is covered with a few layers of keratinized epidermal cells except where penetrated by the pore of its single taste bud (figs. 2, 6D). In histological sections of the tongue the fungiform papillae are most readily recognized by the abundant intradermal neural plexus and the presence of a taste bud. Denervated papillae, lacking nerve fibers and taste buds, are less easily discriminated from the remaining epithelium. In the gerbil the bristle-like filiform papillae surrounding the fungiform papillae occur rather inconspicuously on the dorsal surface of the anterior two-thirds of the tongue. They are most prominent on the dorsal intermolar eminence, an elevated portion of the tongue epithelium which lacks taste papillae.

Taste buds were observed in the buccal
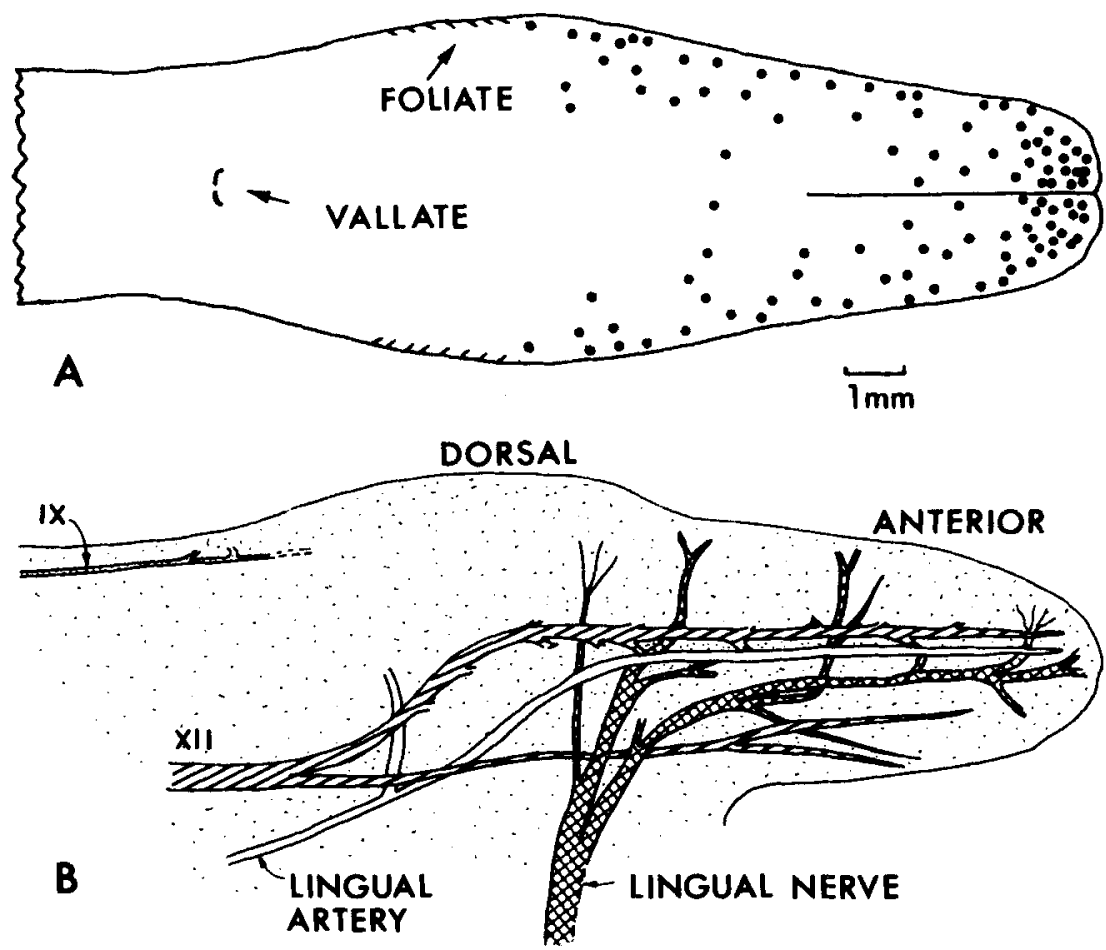

Fig. 1 Drawings of gerbil tongue. The dimensions are correct for fresh tissue. A. This is a schematic drawing of the dorsal surface of the tongue with fungiform papillae indicated by black dots. B. This sagittal projection is based upon fresh tissue dissections and shows pathways of the lingual artery and major nerve trunks [glossopharyngeal (IXth), hypoglossal (XIIth), and lingual]. 
epithelium adjacent to the lateral margin of the tongue, both at the level of the foliate papillae and also at more anterior buccal locations. Taste buds in these locations in mammals have not been previously observed.

The innervation of the tongue is depicted in a sagittal projection based on drawings of fresh tissue dissections of 6 tongues (fig. 1B). The chorda tympani nerve at this level is combined with the lingual nerve proper. The lingual nerve enters the middle of the tongue and trifurcates into posterior, medial, and a more ventral anterior branch which extends to the tip of the tongue. Similar branches have been described for the cat tongue (Ishiko, '74). The hypoglossal nerve (XIIth) enters the tongue ventrally and posteriorly. It traverses the entire length of the tongue as it sends out branches to the intrinsic tongue muscles. One major branch continues ventrally and deviates laterally. The glossopharyngeal nerve (IXth) enters the tongue dorsally and travels directly to the area of the vallate papilla. Some of its fibers extend laterally to the foliate papillae.

These observations of nerve branches seen in fresh tissue dissections were extended by light microscopic examination of serial sagittal sections of 12 silver stained tongues in which selective unilateral surgery left intact one or none of the IXth, XIIth, lingual, and chorda tympani nerves. From these experiments it was determined that: (i) the chorda tympani nerve innervates the fungiform papillae (figs. 4A,B); (ii) the IXth nerve innervates the foliate and vallate papillae and adjacent areas; (iii) the lingual nerve proper innervates the anterior portion of the tongue epithelium including the fungiform papillae.

The source of axons which specifically innervate and maintain taste buds was determined by unilaterally removing either or both the chorda tympani and IXth nerves in 15 animals and examining the tongue for taste buds with Heidenhain's iron hematoxylin stain. We found that fungiform taste buds were maintained only when the chorda tympani was intact. Selective removal of contralateral nerves revealed that, with the possible exception of a few fungiform papillae on or adjacent to the midline, all taste bud innervation in the foliate and fungiform papillae is ipsilateral.

The main axis of fungiform taste buds in gerbils is sometimes tilted rather than normal to the papilla surface. In a mature taste bud many elongated fusiform cells extend from the taste pore to the base of the bud. The nuclei are located in the lower twothirds of the bud. The cells tend to be arranged in parallel thereby defining the main axis of the bud (fig. 6D). In exceptional cases the Winkelman silver stain revealed not only the intradermal neural plexus but also the taste bud cells. In figure 2 chorda tympani and lingual fibers can be seen as they penetrate into the center of the papilla's dermal core (light region) and embrace the taste bud in a chalice-like axonal network. Figures $4 \mathrm{~A}$ and $4 \mathrm{~B}$ show examples of chorda tympani innervation of fungiform papillae in the absence of lingual nerve fibers. It can be seen that numerous axons are arrayed in a complex matrix at the base of the taste bud. Our denervation experiments suggest that the lingual nerve proper lacks taste fibers, since it failed to maintain taste buds in the absence of the chorda tympani nerve.

In the tongue the lingual nerve trunk is densely packed with axons from both the chorda tympani and the lingual nerve proper (fig. $3 \mathrm{C}$ ). The chorda tympani nerve fibers are separately revealed in the common nerve trunk by cutting the lingual nerve proper (fig. 3B). When the chorda tympani is also cut this remaining set of fibers disappears (fig. 3A).

In summary, our background investigations of normal innervation, in preparation for examining regeneration, revealed that the taste fibers of the chorda tympani nerve travel in the combined chorda tympani-lingual nerve trunk and innervate fungiform taste buds. The lingual nerve proper supplies the fungiform papillae with an extensive network of non-taste 


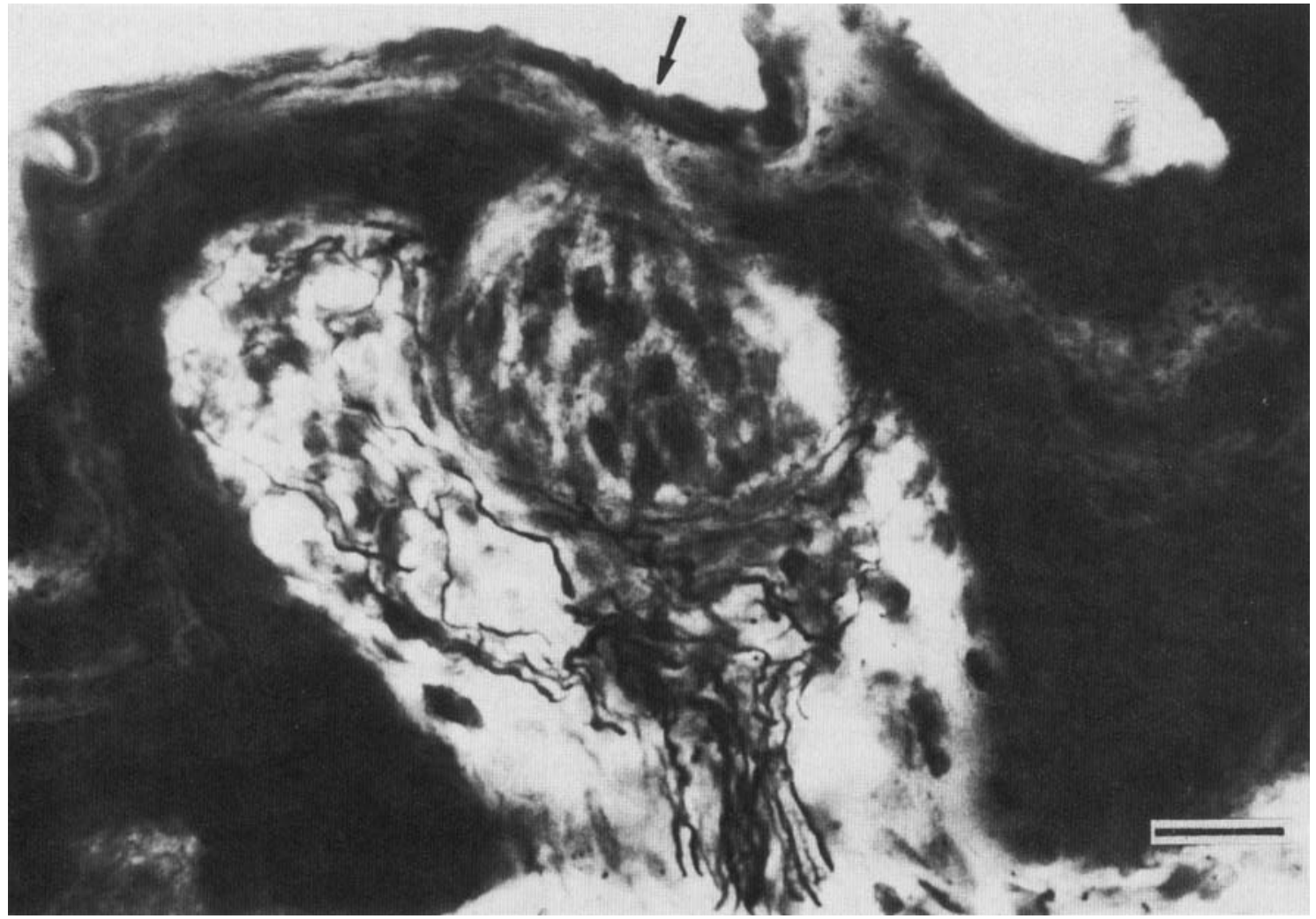

Fig. 2 Fungiform papilla, Winkelman's silver stain. A bundle of axons ascends through the center of the dermal core (light region) to the base of the taste bud. The arrow indicates the taste pore region where chemical substances stimulate the receptors. To the right of the arrow is a portion of a filiform papilla protruding upward. Scale, $20 \mu \mathrm{m}$; section thickness, $25 \mu \mathrm{m}$.

fibers. In the present research on regeneration we have concentrated our attention upon the nerve supply and taste buds in the anterior $10 \mathrm{~mm}$ of the tongue. The area includes virtually all of the fungiform taste buds.

\section{Time course of regeneration}

To determine the temporal sequence of regrowth of crushed chorda tympani fibers in the tongue nerve trunks and in fungiform papillae, it was first necessary to eliminate lingual fibers by transecting the lingual nerve proper. The chorda tympani nerve was then crushed near its junction with the lingual nerve. Electrophysiological recording proximal to the crush indicated that the crushing procedure had functionally interrupted the axons. Evoked and spontaneous activity were eliminated.
The chorda tympani fibers rapidly regenerated into the tongue. They grew within the original chorda tympani-lingual nerve trunks among densely stained remnants of degenerated lingual and chorda tympani axons (fig. 3A). After following the old nerve trunks, the regenerating chorda tympani axons ascended to the superficial tissue to innervate fungiform papillae in the dorsal epithelium. An example is shown

Fig. 3 Nerve trunks within the gerbil tongue, Winkelman's silver stain. A. Chorda tympani-lingual nerve trunk seven days after crushing the chorda tympani and removing portions of the ipsilateral lingual nerve proper, the IXth, and XIIth nerves. Degenerated axonal fragments and a regenerating axon are visible. B. Normal chorda tympani fibers ten days after transecting the ipsilateral lingual, IXth, and XIIth nerves. C. Normal combined chorda tympani-lingual nerve trunk. Scales, $40 \mu \mathrm{m}$; section thickness, $35 \mu \mathrm{m}$. 

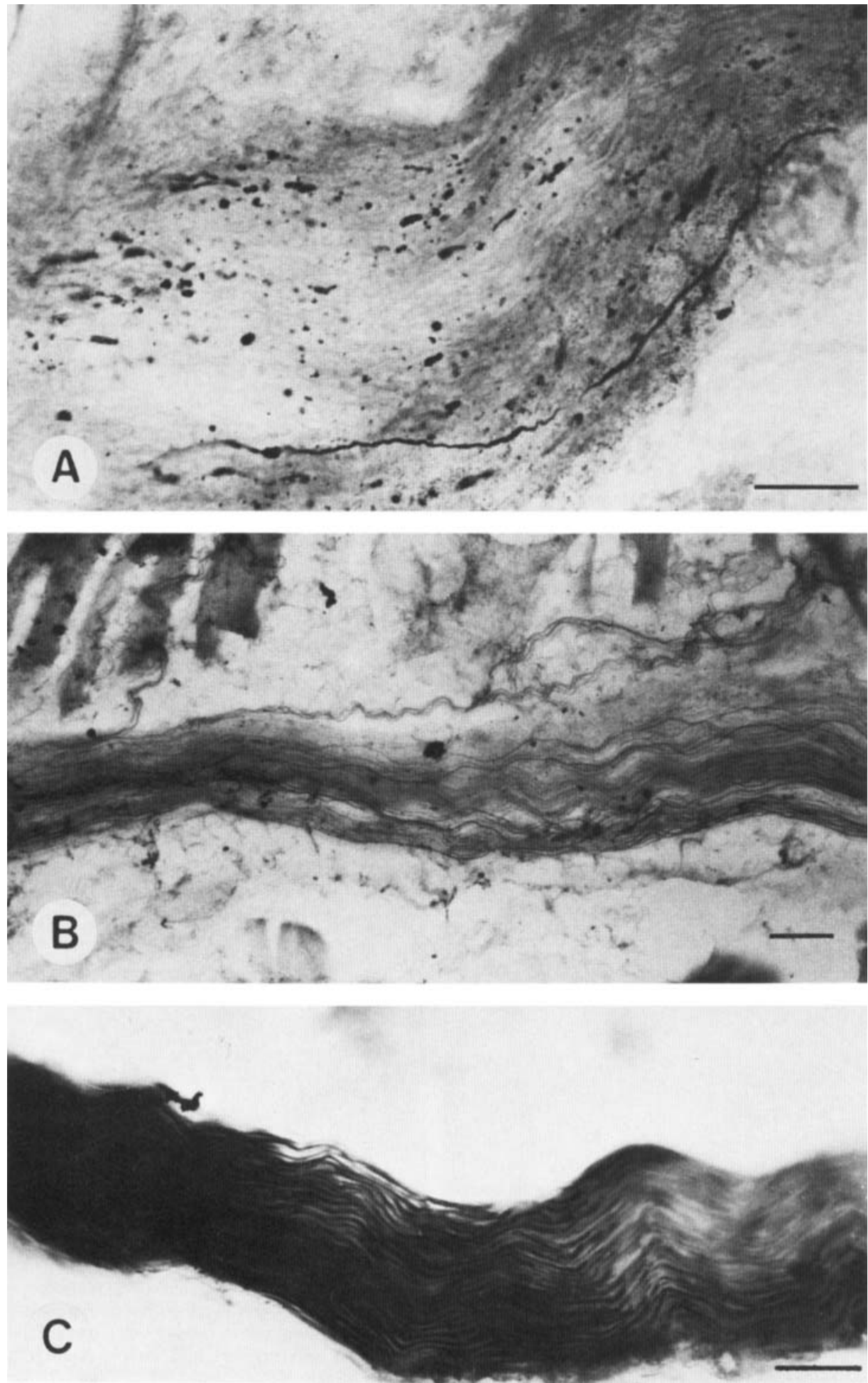

Figure 3 

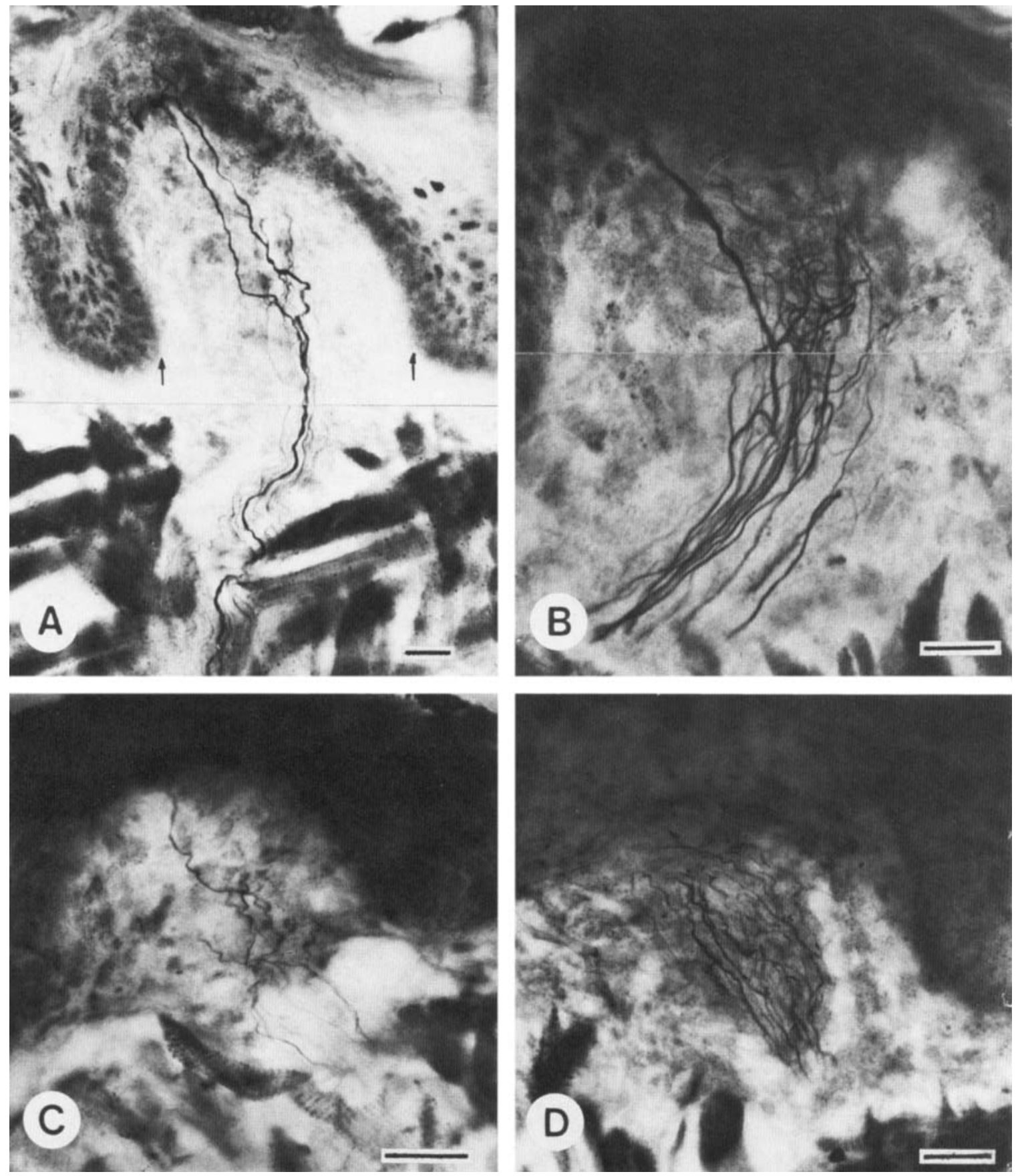

Fig. 4 Fungiform papillae, Winkelman's silver stain. A,B. Photomicrographs of fibers of the normal chorda tympani nerve within fungiform papillae. The ipsilateral IXth, XIIth, and lingual nerve proper had been transected ten days earlier. The arrows in A indicate the diameter of the papilla's dermal core. C. Regenerating chorda tympani fibers ten days after the chorda tympani nerve was crushed. The ipsilateral IXth and lingual nerve proper were transected. D. Regenerating chorda tympani fibers 11 days after the chorda tympani nerve had been crushed and the ipsilateral lingual, IXth, and XIIth nerves transected. Scales, $20 \mu \mathrm{m}$; section thickness A, B, D, $35 \mu \mathrm{m}, \mathrm{C}, 50 \mu \mathrm{m}$. 
in figure $4 \mathrm{C}$ of a fungiform papilla reinnervated 10 days after the chorda tympani had been crushed. As regeneration progressed the number of fibers increased in the papillae (e.g., fig. 4D, 11 days).

To quantify the time course of reinnervation of fungiform papillae we counted the number of fungiform papillae that were reinnervated 7 to 11 days after unilaterally crushing the chorda tympani in 12 gerbils. In all animals the ipsilateral lingual nerve proper and IXth nerve were transected. In half of the animals the ipsilateral XIIth nerve was also transected. There was a systematic increase over time in the percentage of fungiform papillae that were reinnervated (triangles in fig. 5). No axons were seen in the epithelium before eight days. Thereafter axons in the epithelium were almost exclusively restricted to the fungiform papillae.

The time course of taste bud regeneration following crushing of the chorda tympani nerve in 24 gerbils is shown by filled circles in figure 5 . The number of regenerated taste buds is given as percentage of the number of taste buds on the normal side of the tongue in the same gerbil.

It has been widely assumed that taste fibers initiate the reformation of taste buds. This is supported by the results in figure 5 which show that the arrival of nerve fibers in the papillae preceded the appearance of taste buds. From the recovery curves in figure 5 we conclude that regenerating axons of the crushed chorda tympani nerve first reinnervate fungiform papillae at about eight days, whereas taste buds are first observed nine or ten days after the same operation. Hence, taste fibers can reestablish new taste buds one to two days after their arrival in fungiform papillae devoid of taste buds. Control experiments indicated: (i) that taste buds did not reform unless chorda tympani fibers were allowed to regenerate and (ii) that the time of first appearance of regenerating chorda tympani fibers in the papillae was not an artifact of staining properties. Regenerating fibers could be stained before their arrival in the papillae (viz., fig. 3A). Physio- logical experiments, which will be reported separately, showed the reformed taste buds were actually functional at these early times. Cutting rather than crushing the chorda tympani nerve led to delayed taste bud regeneration (open circles, fig. 5 , $n=9$ ).

Denervated fungiform taste buds did not degenerate as rapidly as the crushed axons. Nevertheless, by nine days in more than $90 \%$ of the denervated fungiform taste buds the fusiform cells had been replaced by squamous epithelial cells. Occasional papillae contained one or two residual fusiform cells. Their cytoplasm tended to be darkly stained (figs. 6A,B). Nuclei were not restricted to the more basal regions of these degenerated taste buds.

The first regenerating taste buds were somewhat abnormal in appearance (11 days, fig. 6C). The fusiform cells were less tightly packed and were bordered by basal cells that were as distinct as the cells within the bud. This gave the appearance of a less abrupt transition than normal from the surrounding squamous epithelium to the taste bud cells. Nuclei were present throughout the bud rather than restricted to the basal two thirds. Those regenerated taste buds which were well-developed by day 11 were not significantly smaller in volume than normal taste buds (matched $t$-test, $t=1.63, \mathrm{n}=21$ pairs). The volume of regenerated taste buds was compared to the volume of normal taste buds from the normal side of the tongue on the same or on an adjacent histological section. The volume was computed as the sum of a hemisphere and a hemiellipsoid.

\section{Spatial patterns of regeneration}

The spatial distribution of fungiform taste buds was measured in the first $10 \mathrm{~mm}$ of 18 normal tongues. Normal taste buds were concentrated at the tip of the tongue and more or less evenly distributed along the middle and more posterior tongue regions (filled circles, fig. 7). By 15 to 19 days after crushing the chorda tympani a mean of $64 \%$ of the taste buds had regenerated, at which time their spatial distribu- 


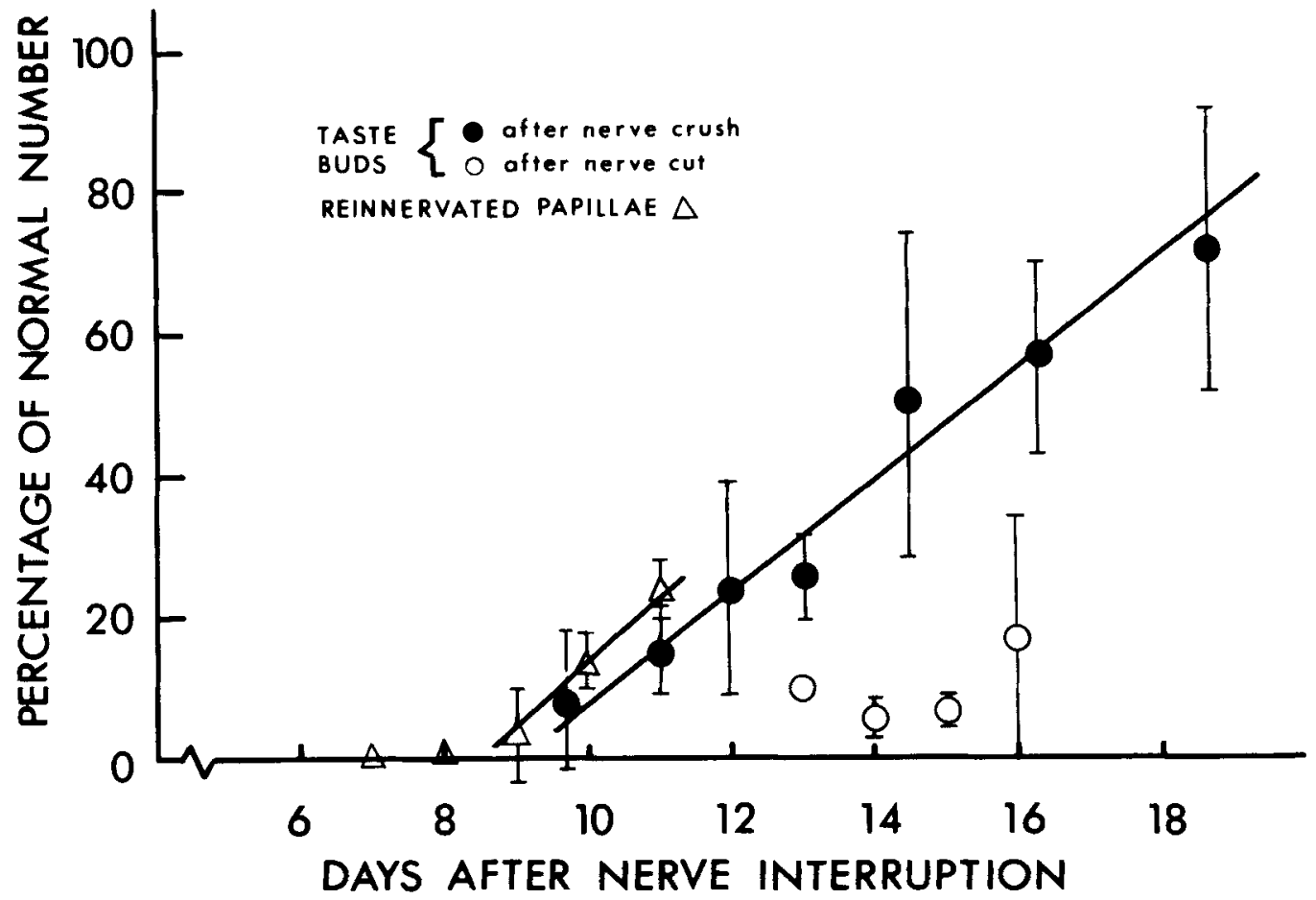

Fig. 5 Time course of reinnervation of fungiform papillae and reformation of taste buds after chorda tympani interruption. Triangles show the mean percentage of fungiform papillae that were reinnervated as a function of days after crushing the chorda tympani. The ipsilateral IXth and lingual nerve proper were cut. $N=12$; two to three animals per data point. (The reference value for the normal number of chorda tympani innervated papillae was the mean $(\bar{x}=53)$ obtained from three control animals in which the IXth, XIIth, and lingual nerves were transected ipsilaterally 10 days prior to silver staining.) Filled circles show the mean number of regenerated taste buds as a percentage of the number of taste buds on the contralateral (normal) side of the same tongue. The chorda tympani had been crushed and in eight gerbils the ipsilateral IXth nerve had also been cut. $\mathrm{N}=24$; three to four animals per data point. (Control experiments in 3 gerbils showed that the left-right differences in number of fungiform taste buds were less than $10 \%$.) Open circles show, as a percentage of the normal side, the mean number of taste buds reformed after transection of the chorda tympani. The ipsilateral IXth nerve was transected and a portion removed. $N=9$; two to three animals per data point, except one at 13 days. Bars indicate standard deviations.

tion (open circles, fig. 7) approximated the normal.

We quantified the spatial distribution of normal chorda tympani and lingual nerve axons by examining serial sections of silver stained tongues. To specify the location of axons, the anterior-posterior length of the dorsal tongue surface of each sagittal section was partitioned into segments $120 \mu \mathrm{m}$ long times the epidermal or dermal thickness (approximately 100 segments per sagittal section). Fibers of the normal chorda tympani nerve were observed in $3.8 \%$ of the epidermal segments (mean of 50 of 1,295 segments, $\mathrm{N}=3$ gerbils; lingual, IXth, and XIIth nerves removed) and $5.2 \%$ of the dermal segments (mean of 66 of 1,303 segments). We concluded that chorda tympani fibers were rarely found in the epithelium apart from fungiform papillae. In contrast, when the common lingual nerve innervated the tongue, axons were readily observed in most of the epidermis ( $76 \%$ of the segments) and dermis ( $98 \%$ of the segments). Many of these lingual fibers terminated in the epithelium; they were 

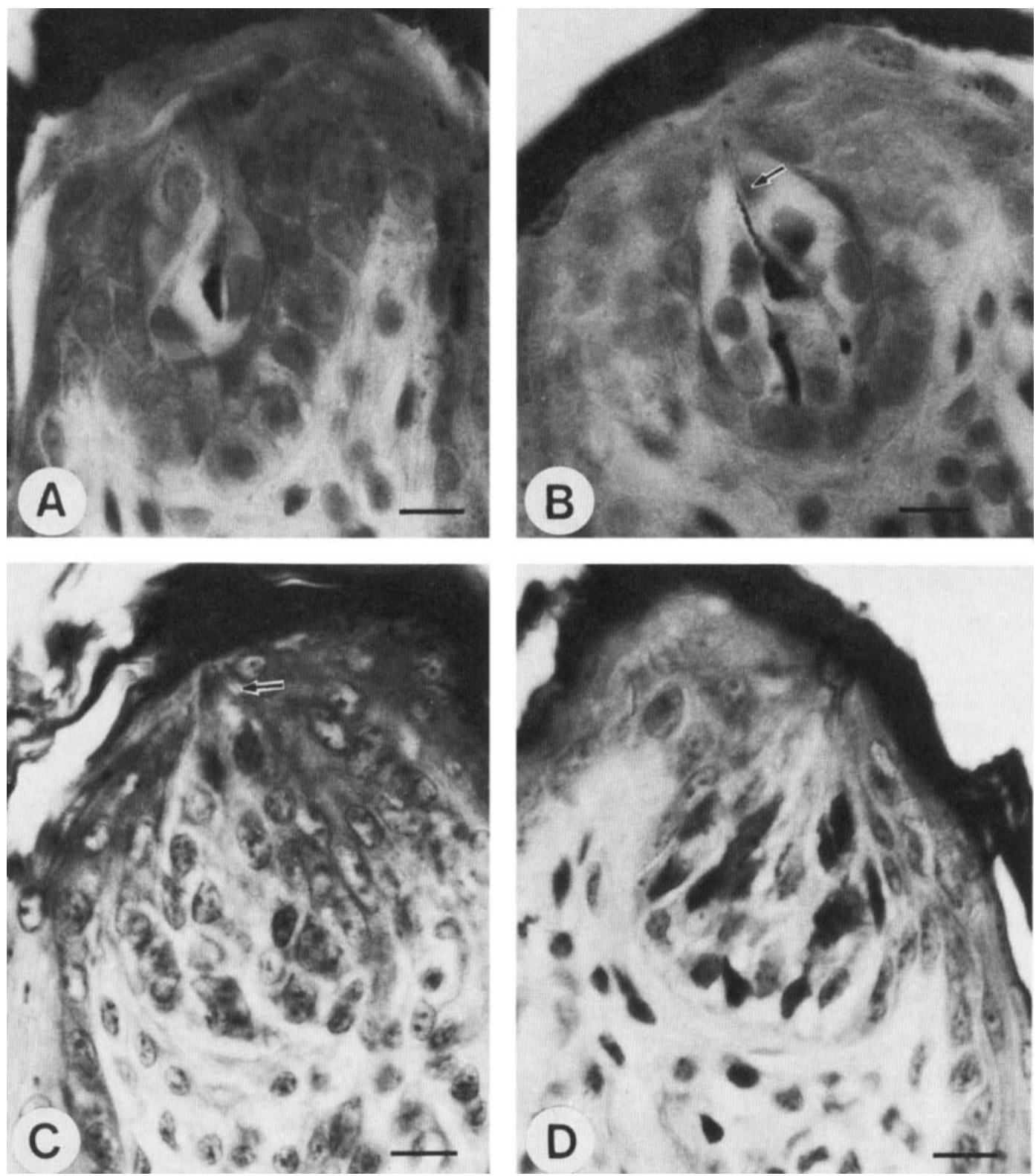

Fig. 6 Fungiform papillae, Heidenhain's iron hematoxylin stain. A,B. Two degenerating taste buds nine days after the chorda tympani and IXth nerve transection. These buds are examples of the best appearing taste buds that could be found at nine days. Most buds had completely degenerated by this time. Note the densely staining material extending far into the neck of a fusiform cell (arrow in B). C. Regenerating taste bud 11 days after crushing of the chorda tympani nerve and transection of the IXth nerve. The arrow indicates the numerous cell processes extending toward the taste pore. D. Normal fungiform taste bud. Scales, $10 \mu \mathrm{m}$; section thickness, $10 \mu \mathrm{m}$. 


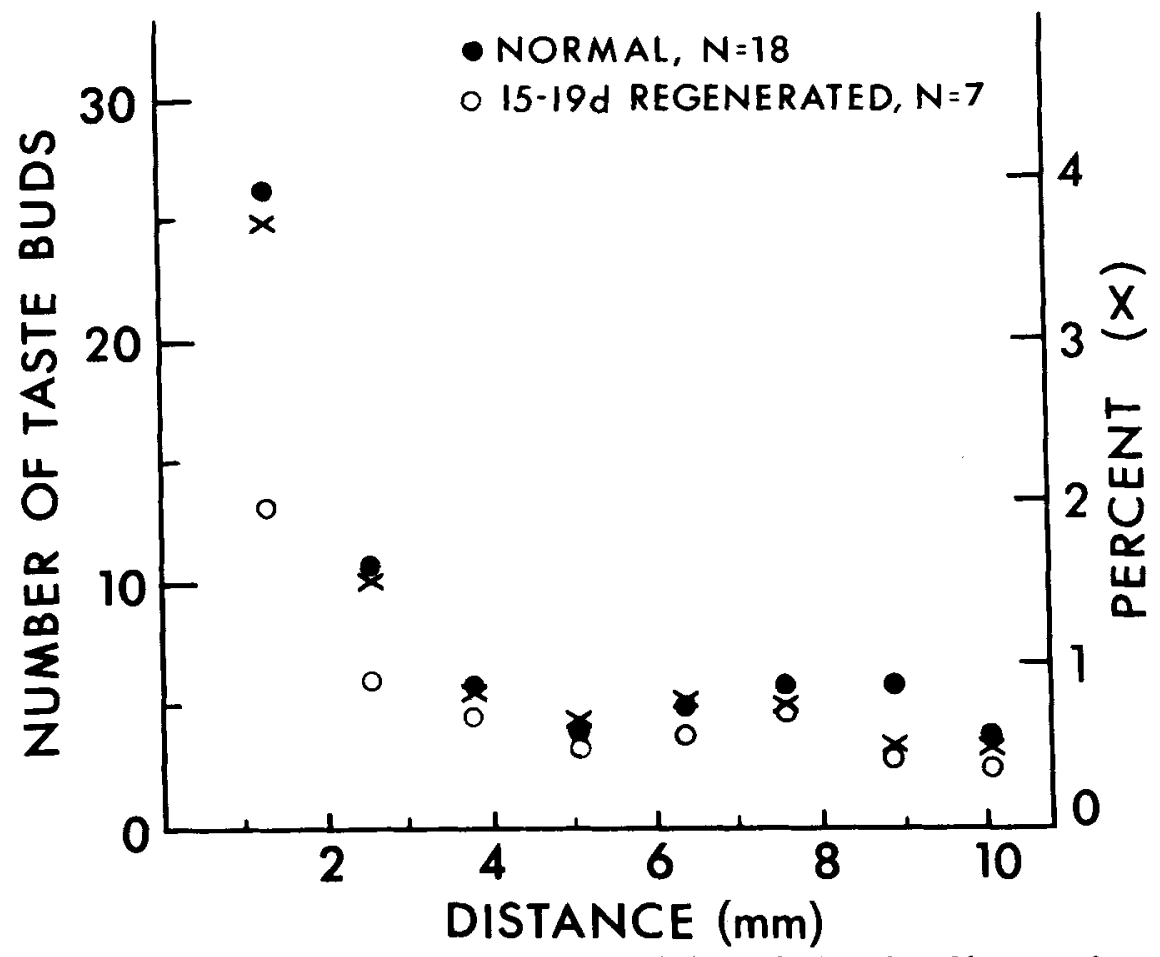

Fig. 7 The number of normal $(-)$ and regenerated $(O)$ taste buds is plotted by units of $1.25 \mathrm{~mm}$ as a function of distance from the tip of the tongue. The total cross-sectional area of the dermal cores of all fungiform papillae is plotted as a percentage of the surface area of the dorsal epithelium of the tongue $(\mathrm{X})$. Dimensions correspond to fresh tissue.

not fibers of passage which took a course parallel to the epithelial surface. We conclude that the normal lingual nerve innervates widespread portions of the tongue epidermis, whereas the chorda tympani nerve is restricted to the fungiform papillae of the epidermis.

In view of these normal differences in lingual and chorda tympani patterns of innervation it was of interest to consider the accuracy of regrowth of regenerating chorda tympani fibers. Each fungiform papilla has a dermal core through which a bundle of chorda tympani axons grows as it ascends rather directly from the underlying muscle layer to the epidermis (figs. $4 A, B)$. In figure $4 A$ the arrows mark the diameter of a dermal core through which axons normally pass to reach taste receptor cell precursors. Taken together these circular areas average about $1 \%$ of the area of the dorsal epidermis (mean cross-sectional dermal core area $\times$ total number of fungiform papillae). The "Xs" in figure 7 show the dermal core area calculated as a percentage of the area of successive $1.25 \mathrm{~mm}$ cross-sectional strips of dorsal epithelium. The strip at the tip of the tongue has the highest percentage because there are more fungiform papillae there. The data in figure 7 indicate that over most of the anterior portion of the tongue dorsum unguided chorda tympani fibers would have had about one chance in 100 of rising upward to the epidermis by penetrating precisely through a fungiform papilla's dermal core.

We examined complete serial sagittal sections of the tongue for regenerating chorda tympani fibers throughout the period of their return to the epidermis. More than $95 \%$ of the $120 \mu \mathrm{m}$ segments which were innervated were associated with 


\section{TASTE BUDS}

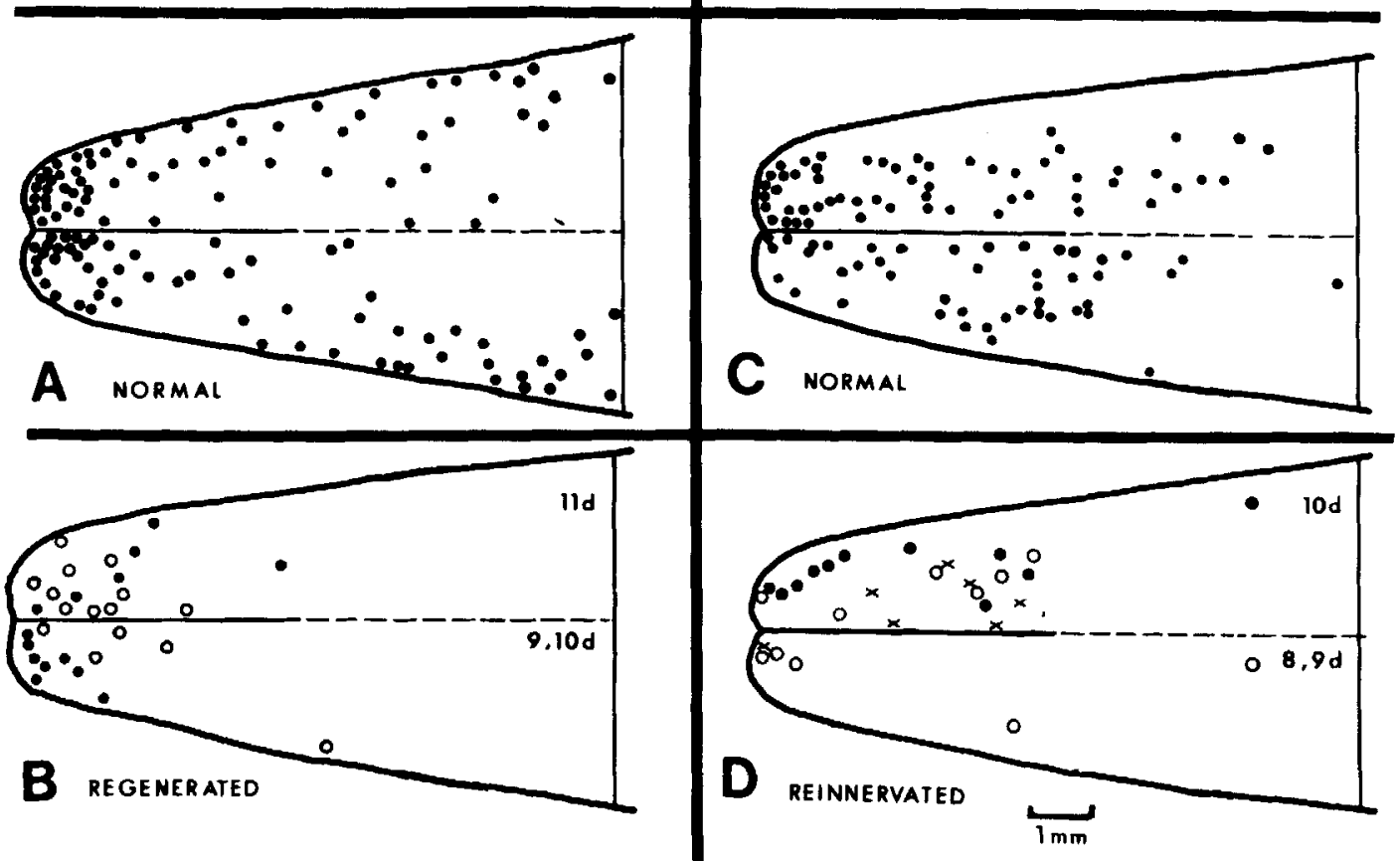

Fig. 8 Dorsal view of the anterior $10 \mathrm{~mm}$ of the gerbil tongue showing the spatial distribution of fungiform taste buds and innervated fungiform papillae. Taste bud distributions were reconstructed from serial $10 \mu \mathrm{m}$ cross-sections. Innervated fungiform papillae distributions were reconstructed from serial $35 \mu \mathrm{m}$ sagittal sections. Dimensions are correct for fresh tissue. A. Example of normal fungiform taste bud distribution of one gerbil. B. Left side of tongue: regenerated taste bud distribution of one gerbil nine days $(O)$ and one gerbil ten days $(-)$ following crushing of the chorda tympani. Right side: two gerbils after 11 days $(O, 0)$. C. Distribution of chorda tympani innervated fungiform papillae, left and right sides of tongue from different control animals. D. Left side of tongue: distribution of reinnervated fungiform papillae of one gerbil eight days $(X)$, and one gerbil nine days $(O)$ following crushing of the chorda tympani. Right side: three gerbils after ten days $(O, \bullet, X)$.

fungiform papillae. Fibers did not stray into lingual axonal pathways and innervate the epidermis between fungiform papillae. Reinnervation, therefore, was highly accurate; it was not a chance phenomenon involving unguided axonal regrowth.

Figure 8 represents data, from the earliest phases of recovery, on the spatial distribution of regenerated fungiform taste buds and reinnervated fungiform papillae. The distribution of fungiform taste buds for one normal gerbil is shown in figure $8 \mathrm{~A}$. Figure $8 \mathrm{C}$ indicates the distribution of normally innervated fungiform papillae, comparing two different gerbils on the left and right sides of the tongue diagram. The spatial distributions of normal taste buds and innervated papillae shown in figures $8 \mathrm{~A}$ and $8 \mathrm{C}$ correspond reasonably well. However, in the silver staining method the delicate sprays of axons were not easily observed at the lateral margins of the tongue because the axons were cut in crosssection or masked by the darker staining keratin of these parasagittal sections.

In figure $8 \mathrm{~B}$ the loci of regenerated fungiform taste buds are indicated on the left side of the tongue for one gerbil at nine days (open circles) and for one gerbil at ten days (filled circles) and on the right side for 
two gerbils at 11 days. The loci of reinnervated papillae after eight days $(\mathrm{n}=1)$, nine days $(n=1)$ and ten days $(n=3)$ of chorda tympani regeneration are shown in figure 8D. Two 7-day, one 8-day, and one 9-day animal had no reinnervated papillae. It is clear that both reinnervated papillae and reformed taste buds were preferentially located in the more anterior portions of the tongue in early stages of regeneration (compare in fig. 8A vs. B and C vs. D). It might be expected that more reformed taste buds would be located in the anterior region simply because there are normally more papillae there. To take into account the non-homogeneous papilla distribution, taste bud locations in each experimental animal were plotted on tongue diagrams such as those in figure 8 . Then, based on the taste bud distribution on the normal side, a gerbil's tongue diagram was divided into four areas, each containing $25 \%$ of the taste buds on the normal side (i.e., divided into antero-posterior quartiles). The null hypotheses was that reformed taste buds would be distributed in accordance with the control taste bud distribution. That is, at all stages of recovery, $25 \%$ of the regenerated taste buds would be found in each quartile area. (The spatial distribution of

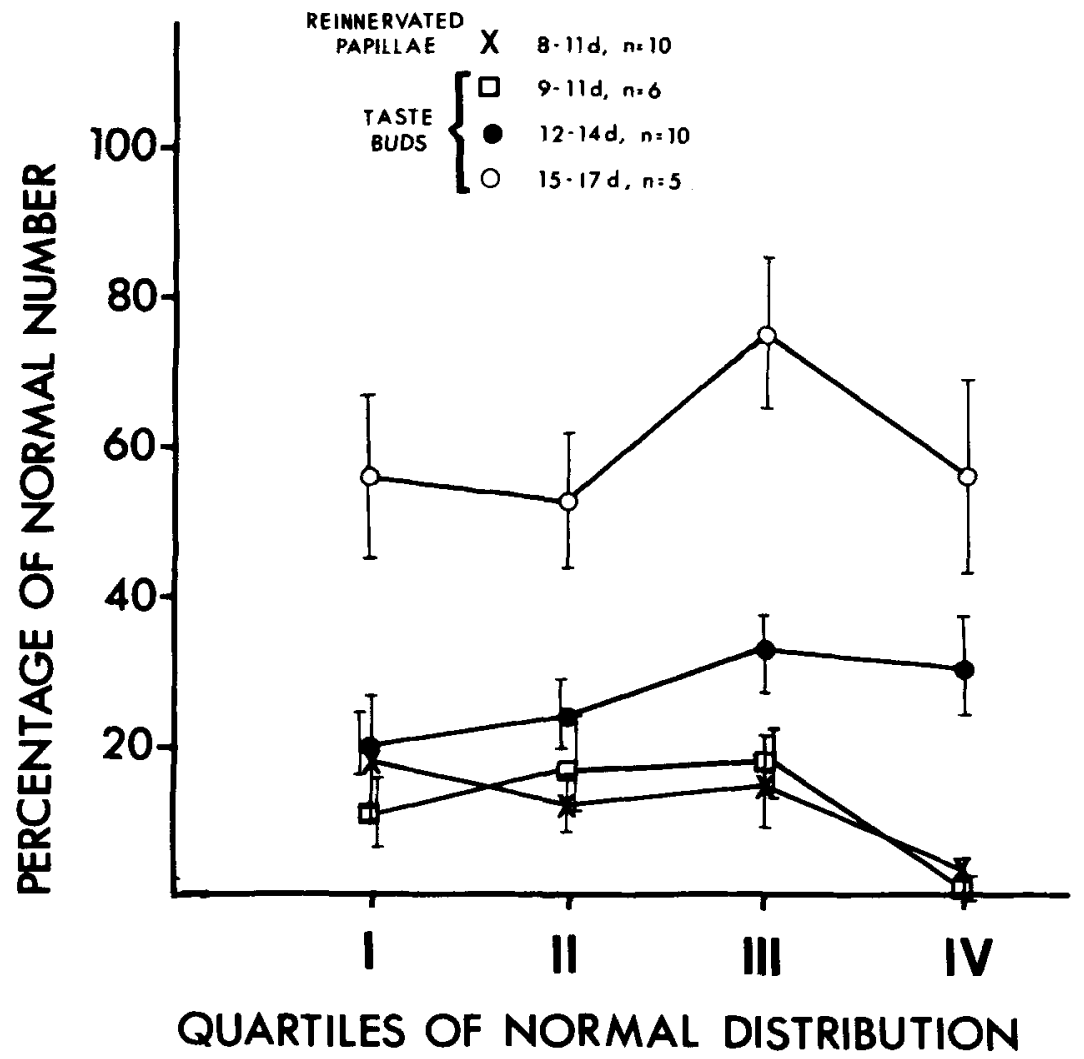

Fig. 9 Spatial distribution of reinnervated fungiform papillae and reformed taste buds in the first $10 \mathrm{~mm}$ of the gerbil tongue. For the analysis of reinnervated papillae the tongue surface of experimental animals was divided into quartiles based on the distribution of innervated papillae in three normal gerbils. Quartile lines were drawn $0.7 \mathrm{~mm}, 2.7 \mathrm{~mm}$, and $4.4 \mathrm{~mm}$ from the tip, marking four areas, each of which contained $25 \%$ or a mean of 13.2 papillae in the normal animals. The number of reinnervated papillae in each quartile was plotted as a percentage of 13.2 papillae. For taste buds the quartile areas were determined for each gerbil from the taste bud distribution on the normal side. Means and standard errors are plotted. 
silver stained reinnervated papillae were evaluated in a similar manner. Quartiles were established from 3 normal tongues.) If taste buds returned in strict proportion to the control distribution, horizontal lines would be obtained in figure 9 for each age group. However, at early stages of recovery, taste buds were significantly underrepresented in the fourth (posterior) quartile ( $p<0.05$, Wilcoxin test, two-tailed). Similarly, reinnervated papillae were also under-represented in the fourth quartile $(p$ $<0.01$, Wilcoxin test, two-tailed). No other deviations from a straight line for any age group were statistically significant $(p$ $>0.05$ ). It is pertinent to point out that the experiments on taste buds and reinnervation of papillae were independent experiments carried out on different animals with different staining procedures. We conclude from figure 9 that regenerating chorda tympani fibers tend preferentially to reinnervate the front of the tongue and generally fail to reinnervate the posterior tongue area in the early stages of reinnervation. At later stages there was no significant difference in spatial pattern from that predicted by the distribution of taste buds in normal gerbils.

Data from figure 8D were used to calculate maximal rates of axonal growth during the regeneration period. After, eight, nine, and ten days of regeneration the most anterior location of silver-stained fibers in the tongue epithelium was measured for each of five gerbils. The mean maximal rate of axon growth was $2.2 \mathrm{~mm} /$ day (Range 1.0$2.6 \mathrm{~mm} /$ day). (The mean distance from the site of chorda tympani crush to the tip of the tongue was $20.7 \mathrm{~mm}$.)

\section{DISCUSSION}

Although the gerbil's tongue is similar to the rat's in gross morphology, types of taste papillae, and distribution of taste buds and their sources of innervation (Whiteside, '27; Fish et al., '44; Oakley, '70; Miller and Preslar, '75), there are some differences: (i) The taste buds of the gerbil are sometimes tilted with respect to the axis of the fungiform papilla. (ii) The rat's fungiform papillae are more distinct and more obviously arrayed in longitudinal rows along the tongue (Miller and Preslar, '75). (iii) Although there are fewer fungiform taste buds in the gerbil $(71 \pm 11$ vs. $93 \pm 9$ S.D. in the rat, unilaterally; Oakley, '70; Miller and Preslar, 75), the density of fungiform papillae is higher in the gerbil because the tongue is smaller: approximately $50 \mathrm{~mm}^{2}$ vs. $200 \mathrm{~mm}^{2}$ in area.

The rate of regrowth of vertebrate taste fibers has not been previously measured. Earlier electrophysiological studies of peripheral nerve regeneration have indicated that the most rapidly regenerating axons grow at rates that are rather similar across types of nerves or species of mammals, but highly dependent upon the time after nerve interruption (e.g., first week, $1.0 \mathrm{~mm}$ / day; second week, $1.9 \mathrm{~mm} /$ day, cat vagus nerve; Guth and Jacobson, '66). In our experiment the fastest growing fibers grew at the rate of $2.2 \mathrm{~mm} /$ day for eight to ten days. This may be an underestimate since some fibers might have terminated at fungiform papillae several hours before sacrifice for histological study.

The rate of nerve regeneration has been shown to be slower after transecting rather than crushing a nerve $(1.6 \mathrm{~mm} /$ day vs. 2.0 $\mathrm{mm} /$ day; Gutmann and Guttmann, '42). In our experiment delayed nerve regeneration after transection of the chorda tympani nerve may have contributed to the delayed reformation of taste buds (fig. 5).

A comparison of the time courses of reinnervation of fungiform papillae and reformation of taste buds (fig. 5) indicated that it requires one to two days after axons have arrived in a papilla to reestablish a taste bud. Such rapid morphological reestablishment of taste buds does not appear to be an artifact of the failure of some taste buds to degenerate. By the time the first taste axons returned to the papillae, most buds had disappeared, as defined by a complete absence of fusiform cells. Without neurotrophic attraction it is unlikely that the first incoming axons would by chance 
locate and reinnervate the few residual fusiform cells. It is more probable that new receptor cells rapidly differentiated under the influence of the reinnervating fibers. (We have used morphological criteria to define a regenerated taste bud. It will be shown in a separate study that physiological recovery of taste responses occurs as early as 11 days.) Fujimoto and Murray ('70) carried out an electronmicroscopic analysis of degenerating and regenerating vallate taste buds in the rabbit. Taste buds degenerated in 10 days. They found no taste buds or axons in the vallate epithelium 21 days after cutting the IXth nerve, yet by the next age examined ( 25 days) there were some nearly fully formed taste buds present. Therefore, in this situation taste fibers must have reestablished taste buds within four days or less after fibers arrived in the vallate epithelium.

Tritiated thymidine labelling of rat fungiform taste buds indicates an average cell life span of eight to twelve days (Beidler and Smallman, '65). Degeneration studies indicate that rat fungiform taste buds degenerate within seven days (Beidler, '63; Farbman, '69). However, recent studies on cell kinetics in rat vallate taste buds indicate that dark cells (presumably receptors) are renewed in approximately six days but that light cells turn over at a much slower rate. They may have a life span of several weeks (A. Farbman, personal communication). If light cells continue to exist in denervated gerbil fungiform taste buds, they would be present in a dedifferentiated form since only a few fusiform cells remain throughout the anterior region of the tongue after nine days of denervation. We cannot rule out the possibility that the rapid reformation of taste buds observed in the present research and the Fujimoto and Murray study may be assisted by the redifferentiation of a residual population of older cells in the taste bud.

The regional distribution of regenerating taste fibers is non-random. Initially more fibers are found at the front of the tongue. Fibers may grow to the front of the tongue faster because the local milieu favors rapid growth in that region, or because those fibers have intrinsically higher regeneration rates.

We have shown in this research that normal chorda tympani fibers terminate in fungiform papillae; lingual nerve fibers terminate throughout the dorsal epithelium. What mechanisms guide regenerating chorda tympani fibers almost exclusively to the fungiform papillae? Hafek and Thomas ('68) found that the collagenous tube surrounding the Schwann cells and the axon remains intact for at least one week following nerve crush. In view of their results and the accurate regeneration of the crushed chorda tympani fibers in our experiment, it is likely that the axonal tubes of the chorda tympani remained intact. This would have provided an excellent guidance mechanism for the return of the regenerating axons to the fungiform papillae.

\section{ACKNOWLEDGMENTS}

We are most grateful to L. Hewitt, S. Igras, and M. Mac for their dedicated assistance in various phases of this research. This work was supported in part by U.S.P.H.S. Grant N.S. -07072.

\section{LITERATURE CITED}

Beidler, L. M. 1963 Dynamics of taste cells. Proc. 1st International Sympos. on Olfaction and Taste. Pergamon Press, N.Y., pp. 133-148.

Beidler, L. M., and R. L. Smallman 1965 Renewal of cells within taste buds. J. Cell. Biol., 27: 263-272.

Conger, A. D., and M. A. Wells 1969 Radiation and aging effect on taste structure and function. Rad. Research, 37: 31-49.

El-Eishi, H. I., and F. A. State 1974 The role of the nerve in the formation and maintenance of taste buds. Acta. Anat., 89: 599-609.

Farbman, A. I. 1969 Fine structure of degenerating taste buds after denervation. J. Embryol. Exp. Morph., 22: 55-68.

Fish, H. S., P. D. Malone and C. P. Richter 1944 The anatomy of the tongue of the domestic Norway rat. I. the skin of the tongue; the various papillae, their number and distribution. Anat. Rec., 89: 429-440.

Fujimoto, S., and R. G. Murray 1970 Fine structure of degeneration and regeneration in denervated rabbit vallate taste buds. Anat. Rec., 168: 393-413.

Guth, L. 1957 The effects of glossopharyngeal nerve transection of the circumvallate papilla of the rat. Anat. Rec., 128: 715-731. 
1958 Taste buds on the cat's circumvallate papilla after reinnervation by glossopharyngeal, vagus and hypoglossal nerves. Anat. Rec., 130: 2537.

Guth, L., and S. Jacobson 1966 The rate of regeneration of the vagus nerve of the cat. Exptl. Neurol., 14: 439-447.

Gutmann, E., and L. Guttmann 1942 Factors affecting recovery of sensory function after nerve lesions. J. Neurol. Psychiat., 5: 117-129.

Haftek, J., and P. K. Thomas 1968 Electron-microscope observations on the effects of localized crush injuries on the connective tissues of peripheral nerve. J. Anat., 103: 233-243.

Ishiko, N. 1974 Local gustatory functions associated with segmental organization of the anterior portion of cat's tongue. Exptl. Neurol., 45: 341-354.

Iwayama, T. 1970 Changes in the cell population of taste buds during degeneration and regeneration of their innervation. Z. Zellforsch., 110: 487-495.

Jeppsson, P. H. 1969 Studies on the structure and innervation of taste buds. Acta Otolaryng. Suppl., 259: 1-95.

Miller, I. J., and A. P. Preslar 1975 Spatial distribution of rat fungiform papillae. Anat. Rec., 181:679684.

Murray, R. G., and A. Murray 1969 Degeneration in taste buds of rabbit foliate papillae after section of the glossopharyngeal nerve. Anat. Rec., 163: 234.
Oakley, B. 1967 Altered temperature and taste responses from cross-regenerated sensory nerves in the rat's tongue. J. Physiol., 188: 353-371.

- 1970 Reformation of taste buds by crossed sensory nerves in the rat's tongue. Acta physiol. Scand., 79: 88-94.

- 1974 On the specification of taste neurons in the rat tongue. Brain Res., 75: 85-96.

Olmsted, J. M. D. 1921 Effect of cutting the lingual nerve of the dog. J. Comp. Neur., 33; 149-155.

State, F. A., and R. E. M. Bowden 1974 The effect of transection of the glossopharyngeal nerve upon the structure, cholinesterase activity and innervation of taste buds in rabbits. J. Anat., 118: 77-100.

Vij, S., R. Kanagasuntheram and A. Krishnamurti 1972 Enzymic changes in taste buds of monkey following transection of glossopharyngeal nerve. J. Anat., 113: 425-432.

Vintschgau, M. V., and J. Hönigschmied 1877 Nervus Glossopharyngeus und Schmeckbecher. Arch. Ges. Physiol., 14: 443-448.

Whiteside, B. 1927 Nerve overlap in the gustatory apparatus of the rat, J. Comp. Neur., 44: 363-377.

Winkelmann, R. K. 1960 Nerve Endings in Normal and Pathologic Skin. C. C. Thomas, Springfield.

Zalewski, A. A. 1969 Combined effects of testerone and motor, sensory, or gustatory nerve reinnervation on the regeneration of taste buds, Exp. Neurol., 24: 285-297. 